\title{
Severity One Out of Four
}

National Cancer Institute

\section{Source}

National Cancer Institute. Severity One Out of Four. NCI Thesaurus. Code C147500.

A severity result of one, on a four level scale. 